\title{
DRG, BSC and EBM: time to work with one another
}

\author{
S Ghaffari ${ }^{*}$, M Abolhallaj ${ }^{2}$, A Ghafouri ${ }^{3}$ \\ From 26th Patient Classification Systems International (PCSI) Working Conference \\ Munich, Germany. 15-18 September 2010
}

\section{Introduction}

There has been much evidence provided on the appropriateness of Diagnosis Related Groups (DRGs) as a useful tool for funding, and/or a reimbursement mechanism. DRGs have also proven valuable for management and review of utilization, as well as in conducting research into health systems. Many people believe that DRG-based funding (and performance) holds hospitals accountable for costs, but its impact on quality of care and health outcomes is unclear. This, in turn, undermines its utilization either for funding or management purposes. If classification of similar patients into the classes of DRGs paves the way toward achieving the objectives of evidence-based practice in medicine and the health system, then this will have a significant effect on the utilization of DRGs around the globe.

\section{Methods}

Review of literature

\section{Results}

The practice of evidence-based medicine (EBM) at the DRG level, and the link between DRGs and evidencebased management, will make the DRG system more of an aid by providing an opportunity to produce clinical guidelines and set policy and regulations. Evidence-based medicine attempts to ensure the best prediction of outcomes in medical treatment. It does this through identification of problems, specification of intervention, and the comparison and evaluation of outcomes of treatments.

It is a possibility that practicing EBM for DRG classes in which homogeneous patients with similar clinical and resource intensity conditions have been classified will make DRGs more popular and useful to physicians and health professionals. Then, they will have less of a tendency to regard

1Iranian Social Security Organisation (Member of the Executive Board of Milad Hospital), Tehran, Iran

Full list of author information is available at the end of the article
DRGs as an invasive tool whose purpose is to control the cost of health services at the price of quality. The classification of a homogeneous patient into a DRG class provides the opportunity to collect strong evidence on the risks and benefits of therapeutic intervention, as aimed at by clinical trials.

On the other hand, the primary purpose for which DRGs were designed - to assist in the management of hospitals and make efficient use of health-care resources can be extended by combining DRGs and the balanced scorecard (BSC) as a strategic performance-management tool. If the cost of the production of a single DRG can influence overall financial and non-financial performance of a hospital, then it would be plausible to implement the BSC in a DRG environment. A manager could then supervise the execution of activities done for treatment of a group of patients within a DRG and monitor the cost and quality of their actions.

\section{Conclusions}

Improving health-care performance and promoting efficient management of health-care resources are the main goals of a health system. As well, they are common goals among DRGs, EBM and BSC. The acceptance and popularity of DRGs, as well as the achievement of their initial objectives, will be facilitated if DRGs, EBM and BSC work with one another.

\section{Author details}

${ }^{1}$ Iranian Social Security Organisation (Member of the Executive Board of Milad Hospital), Tehran, Iran. ${ }^{2}$ Budgeting, Ministry of Health, Tehran, Iran. ${ }^{3}$ Management School, Iran University of Medical Science, Tehran, Iran.

Published: 6 October 2010

doi:10.1186/1472-6963-10-S2-A4

Cite this article as: Ghaffari et al:: DRG, BSC and EBM: time to work with one another. BMC Health Services Research 2010 10(Suppl 2):A4. 